\title{
Deshidratación osmótica de mango (mangifera indica). Aplicación al escarchado
}

\author{
Germán Antonio Giraldo G.* \\ Amparo Chiralt B.** \\ Pedro Fito $M^{* *}$
}

* Ph.D. - Profesor - Facultad de Ingeniería - Universidad del Quindío - Armenia, Colombia

E-mail:ggiraldo@uniquindio.edu.co

** Ph.D. Profesores - Universidad Politécnica de Valencia, España

Fecha de recepción: Enero 18 de 2005

Fecha de aprobación: Junio 10 de 2005

\section{RESUMEN}

En esta investigación se estudió la cinética y equilibrado del mango durante la deshidratación osmótica a presión atmosférica, con y sin pulso a vacío, usando soluciones de 35, 45, 55 y $65^{\circ}$ Brix. La cinética se determinó en tiempos cortos, mientras que el equilibrado se realizó en tiempos largos. En ambos casos se analizó la ganancia de azúcar, pérdida de agua, variaciones de masa y volumen de las muestras. Para alcanzar el escarchado, se continuó el proceso con secado por aire caliente, hasta alcanzar concentraciones de 68 y $72^{\circ}$ Brix. Se evaluaron los cambios composicionales, además del análisis textural y sensorial en los productos finales. Las muestras 
$2565^{\circ}$ Brix y $4545^{\circ}$ Brix presentaron las menores variaciones de masa y volumen y mejor textura al final del secado.

Palabras clave: Mango, Cinética de deshidratación, Escarchado, Secado.

\section{ABSTRACT}

In this investigation the kinetics and equilibrium was studied of the mango during the osmodehydration to atmospheric pressure, with and without pulse to vacuum, using solutions of $35,45,55$ and $65^{\circ}$ Brix. The kinetics was determined in short times, while the equilibrium was carried out in long times. In the process it was analyzed the gain of sugar, loss of water, variations of mass and volume of the samples. To reach the candying, you continuous the process with having dried off by hot air, until reaching concentrations of 68 and $72^{\circ}$ Brix. The changes composicionales was evaluated, besides the textural and sensorial analysis in the final products. The samples $2565^{\circ} \mathrm{Brix}$ and $4545^{\circ} \mathrm{Brix}$ showed the smallest variations of mass and volume and better texture at the end of the drying.

Key words: mango, dehydration kinetics, drying, candying.

\section{INTRODUCCIÓN}

El mango es una fruta con una alta producción mundial (FAO, 2000), sin embargo, apenas es comercializado a pesar de la calidad producida, debido a las dificultades en el manejo poscosecha en los países productores. Ésta es una de las frutas más apreciadas por los consumidores, no solamente como producto fresco, sino como ingrediente en helados, ensalada de frutas o snack.

La deshidratación osmótica a temperatura suave, puede ser una tecnología adecuada para el procesamiento de frutas, como el mango, ya que ayuda a mantener el flavor y otras propiedades sensoriales en el producto. Los tratamientos osmóticos, con aplicación de vacío al inicio del proceso (DOPV), pueden producir efectos benéficos en la cinética del proceso y en la calidad de las frutas (Fito P. \& Chiralt A. 2000), debido a que se extrae de la fruta el gas de los poros y se remplaza con el fluido externo (Fito $P$. et al., 1996). La concentración de la solución osmótica afecta directamente la respuesta a la impregnación a vacío (IV) (Giraldo G. et al., 2003; Barat J., Fito P., \& Chiralt A. 2001a), lo que influye en las características finales del producto. La sacarosa es el azúcar mayoritario del mango (Gil A. et al., 2000) y por eso su uso en deshidratación osmótica (DO), busca conferirle pocos cambios a las propiedades sensoriales.

El proceso de DO pasa por varios estados de seudoequilibrio; en el primero de ellos la velocidad del flujo del agua desde el alimento hacia la solución concentrada es mayor que la entrada de sólidos hacia el interior del alimento (Barat J., Fito P., \& Chiralt A. 2001b). Al final de los seudoequilibrios se alcanza la igualdad de la actividad del agua $\left(a_{w}\right)$ del alimento y de la solución osmótica (Argaiz A. et al.,. 1994; Vial C., Gulibert S. \& Cuq J. 1991). El uso IV afecta al equilibrado osmótico, debido a que contribuye a los cambios en la composición y evolución de la masa de la muestra, además, la mayor viscosidad de la solución osmótica, produce un menor grado de impregnación en el equilibrio, aunque se puede incrementar con la IV (Giraldo G. et al., 2003). Diferentes autores reportan algunos resultados del equilibrado osmótico en frutas, por ejemplo: el mango en una disolución de $30^{\circ}$ Brix, alcanzó el equilibrado osmótico a las 48 h. La piña alcanzó el equilibrio a las 24 h de tratamiento con pulso a vacío y 48 h con presión atmosférica (Barat J. et al.,. 2000), la fresa lo alcanzó en un periodo entre 72 y 96 h, mientras que en el Kiwi se alcanzó entre 48 y 72 h (Talens P. 2002).

En la conservación de frutas, el secado combinado juega un papel importante en el 
combinado juega un papel importante en el desarrollo de nuevos productos. La DO, como pretratamiento $(\mathrm{PO})$, proceso que favorece la disminución de los cambios adversos que ocurren durante el uso de métodos combinados, mientras que el secado ayuda a conservar por más tiempo la fruta. Entre los trabajos más relevantes que utilizó el PO en el deshidratado de frutas, se encuentra el realizado por Barat et al., (2002), en escarchado de piña por métodos no térmicos. Mújica et al., (2000) encontraron que se favorece la pérdida de agua al incrementar la concentración de la solución osmótica en procesos con pulso a vacío. Ribeiro \& Anuncio (2000) concluyeron que la convección externa es la que controla el transporte térmico, y la difusión interna del agua es el mecanismo que controla la transferencia total. Maldonado \& Samman (2000) escaldaron y deshidrataron mango en una disolución de azúcar de $60^{\circ}$ Brix por 6 horas y posteriormente lo secaron en estufa con circulación de aire; los resultados fueron muy apreciados y considerados como una alternativa tecnológica para la conservación del mango.

En este trabajo se analizó el efecto causado por el secado, combinando la deshidratación osmótica con y sin aplicación de pulso a vacío, en soluciones de sacarosa de diferente concentración, y el secado con aire caliente, en la calidad en el mango escarchado, lo que ayudo a determinar algunas de las variables del rendimiento y de las propiedades sensoriales.

\section{MATERIALESY MÉTODOS}

\section{Preparación de la muestra}

Los mangos (var Kent) fueron comprados en un mercado local, con un grado de madurez similar, se pelaron y se cortaron en mitades, se extrajeron cilindros de $1.5 \mathrm{~cm}$ de alto y $2 \mathrm{~cm}$ de diámetro en dirección al hueso y se tomaron muestras por triplicado a cada tiempo para la caracterización de la composición (humedad y sólidos solubles).

\section{Tratamientos}

Osmótico: Las muestras de mango fueron sumergidas en soluciones de sacarosa de 65, 55, 45 y $35^{\circ}$ Brix a $30^{\circ} \mathrm{C}$. Se realizaron dos tipos de tratamientos (DO y DOPV). A los 15, 30, 45, 60, 180 y 300 min se tomaron muestras de cada tratamiento y se les determinó la masa $(M)$, el volumen $(V)$, la humedad $\left(x_{w}\right)$ y los sólidos $\operatorname{solubles}\left(x_{\mathrm{ss}}\right)$.

Equilibrado: El estudio se realizó en botes de vidrio con cierre hermético. En ellos se colocaron soluciones osmóticas de sacarosa a $65,55,45$ o $35{ }^{\circ}$ Brix a $30^{\circ} \mathrm{C}$, se trabajó por triplicado, se llevaron igualmente los dos tipos de tratamientos. El muestreo se realizó a las 5, 24, 48, 72, 144, 240 y 720 horas, a cada muestra se le determinó humedad, sólidos solubles y actividad de agua $\left(a_{w}\right)$, asícomo masa y volumen.

Escarchado: El estudio se inició con el PO, en dos periodos: El primer periodo se compuso de IV (a 50 mbar los primeros $10 \mathrm{~min}$ ), y presión atmosférica por 20 min más, usando soluciones osmóticas (5O) a 25, 35, 45, 55 y $65^{\circ}$ Brix, y en el segundo periodo las muestras se llevaron a 65ํㅏㅅ por 72 horas para su equilibrado. Adicionalmente se realizó una serie experimental con $45^{\circ}$ Brix todo el tiempo. Al final del proceso se analizó $x_{w}, x_{s s}$ y $\Delta M$. Los cilindros deshidratados osmóticamente se secaron por corriente de aire a $35^{\circ} \mathrm{C}$, hasta fracciones másicas de sólidos solubles de la fase líquida $\left(z_{s}\right)$ de 0,68 y 0,72 . Con estos y los valores iniciales de humedad y sólidos solubles se estimó la variación de masa en la etapa de secado. Las muestras secadas se sometieron a ensayos de corte, análisis de $x_{w}, x_{s s} y$ M. A partir de los datos obtenidos se calcularon las variaciones de masa $(\Delta M)$, agua $\left(\Delta M_{w}\right)$ y solutos $\left(\Delta M_{s}\right)$ y la variación de volumen $(\Delta V)$.

\section{Pruebas sensoriales}

Se realizaron dos pruebas de evaluación sensorial (dureza), una primera comparó la muestra elaborada por el método tradicional y que se 
llamó comercial, con las muestras deshidratadas osmóticamente por 72 horas en dos etapas de proceso y luego secadas, así: 45 y 45, 35 y 65, 55 y 65 y 65 y 65 . La segunda comparó las muestras deshidratadas osmóticamente por 72 horas y luego secadas, así: 45 y 45 con 25 y 65 , 45 y 65 y 65 y 65 . La evaluación consistió en una prueba escalar de siete puntos, clasificando las muestras presentadas de menor (-) a mayor (+) intensidad del atributo correspondiente con relación a una muestra de referencia. Cada juez evaluó tres veces cada tratamiento en función de la textura.

\section{Determinaciones analíticas}

El contenido de humedad fue obtenido por el método de secado 20.013 a $60^{\circ} \mathrm{C}$ hasta masa constante (A.O.A.C. 1980) y los sólidos solubles usando un refractómetro. (ABBE ATAGO 89553 de Zeiss) El volumen de las muestras se determinó en un picnómetro, por desplazamiento del volumen usando como líquido de referencia la solución isotónica correspondiente y la actividad del agua (aw) en un equipo de punto de rocío, Decagón. Los ensayos mecánicos de corte se realizaron en un texturómetro Stable Micro Systems TA.XT2, con la célula de corte Warner Bratzler HDP/BS, a una velocidad de $10 \mathrm{~mm} / \mathrm{s}$.

\section{RESULTADOS Y DISCUSIÓN}

Los valores de humedad y sólidos solubles determinados en las muestras frescas fueron de $0.826 \pm 0.014$ y $0.156 \pm 0.016$ respectivamente.

\section{Estudio cinético (variaciones de masa y volumen)}

La variación de masa $(\Delta M)$, ecuación 1 y de volumen $(\Delta V)$, ecuación 2 , ha sido modelizada, graficando $\Delta M$ y $\Delta V$ en función de la raíz cuadrada del tiempo (Fito \& Chiralt, 1997). De los gráficos realizados se obtuvieron los valores de la fracción másica de la disolución $\left(\mathrm{y}_{\mathrm{s}}\right)$, la ordenada en el origen $\left(k_{0}\right)$ y la pendiente $(k)$ de cada recta, que se presentan en la tabla 1. También se muestra el valor de $r^{2}$ coeficiente de correlación para cada ecuación lineal obtenida. Los valores presentados de cada variable son los obtenidos transcurridos 5 h de tratamiento.

$$
\begin{aligned}
& \Delta \mathrm{M}=\frac{\mathrm{M}^{\mathrm{t}}-\mathrm{M}^{0}}{\mathrm{M}^{0}} \\
& \Delta \mathrm{V}=\frac{\mathrm{V}^{\mathrm{t}}-\mathrm{V}^{0}}{\mathrm{~V}^{0}}
\end{aligned}
$$

Donde:

$M^{t}=$ Masa tiempot

$M^{0}=$ Masa tiempo inicial

$\mathrm{V}^{\mathrm{t}}=$ Volumen tiempo t

$V^{0}=$ Volumen tiempo inicial

Tabla 1. Parámetros cinéticos de las variaciones de masa $(\Delta M)$ y volumen $(\Delta V)$ en función de la raíz cuadrada del tiempo(Regresión $k=$ pendiente; $k^{\circ}=$ intercepción $y$

\begin{tabular}{|c|c|c|c|c|c|c|c|c|c|}
\hline & ys & $k^{\circ} M \times 10^{2}$ & $\begin{array}{c}k M \times 10^{2} \\
\left(s^{-0.5}\right)\end{array}$ & $R^{2}$ & $\begin{array}{c}\Delta M \times 10^{2} \\
(5 \mathrm{~h})\end{array}$ & $k^{\circ} V \times 10^{2}$ & $\begin{array}{l}k V \times 10^{2} \\
\left(s^{-0.5}\right)\end{array}$ & $R^{2}$ & $\begin{array}{c}\Delta V \times 10^{2} \\
(5 h)\end{array}$ \\
\hline \multirow{4}{*}{$D O$} & 0.65 & 0 & -0.29 & 0.989 & -39.7 & 0 & -0.27 & 0.993 & -37.6 \\
\hline & 0.55 & 0 & -0.32 & 0.968 & -42.3 & 0 & -0.27 & 0.972 & -34.8 \\
\hline & 0.45 & 3.01 & -0.19 & 0.989 & -23.8 & 4.15 & -0.22 & 0.992 & -29.7 \\
\hline & 0.35 & 2.02 & -0.12 & 0.998 & -14.6 & 3.36 & -0.16 & 0.990 & -18.7 \\
\hline \multirow{4}{*}{$D O P V$} & 0.65 & -4.13 & -0.23 & 0.977 & -36.5 & -9.54 & -0.29 & 0.994 & -47.8 \\
\hline & 0.55 & 3.22 & -0.19 & 0.965 & -22.6 & -4.02 & -0.21 & 0.997 & -31.4 \\
\hline & 0.45 & 7.94 & -0.05 & 0.700 & 0.96 & 2.21 & -0.08 & 0.999 & -8.7 \\
\hline & 0.35 & -1.38 & -0.04 & 0.839 & -8.2 & -6.71 & -0.07 & 0.964 & -15.3 \\
\hline
\end{tabular}
$r^{2}=$ coeficiente de correlación)

Es notable la ganancia de masa ocurrida en el tratamiento DOPV a $45^{\circ}$ Brix después del pulso a vacío. Esta ganancia puede atribuirse al efecto combinado de la concentración y la viscosidad en la solución osmótica que incrementa la impregnación efectiva de la muestra, promoviendo la deshidratación rápida de la célula y la difusión de solutos a través de los poros. 
En los tratamientos DOPV con aplicación de pulso a vacío al principio del proceso, el efecto de la concentración de la SO, presentó diferencias en los flujos de agua y solutos. La constante ( $k$ ) de la variación de masa mostró diferencias notables, entre DO y DOPV, debido a los cambios estructurales inducidos por el pulso a vacío, lo que altera las propiedades de los tejidos a la transferencia de masa.

En la figura 1 se aprecia la relación entre la ganancia de azúcar y la pérdida de agua ( M $_{\text {J }} / \mathrm{AM}_{\mathrm{w}}$ ) obtenida en cada tratamiento, en función del tiempo de proceso. La influencia del tiempo de proceso es máxima al inicio del mismo. Los valores asintóticos de $A M_{s} / A M_{w}$ se ven incrementados a medida que se disminuye la concentración osmótica y se aplica el pulso a vacío, lo que muestra valores excepcionalmente elevados para el tratamiento DOPV a $45^{\circ}$ Brix. Este comportamiento indica que la difusión está acoplada con la acción de mecanismos hidrodinámicos al inicio del proceso.
Se ajustó a la ecuación fickiana simplificada (un único término de la solución seriada para tiempos cortos) para un cilindro finito, donde $r$ es el radio del cilindro y I la altura del cilindro (Crank, J. 1975).

$1-Y=1.128\left(\frac{2}{r}+\frac{1}{l}\right) \sqrt{D_{e} t}$

Tabla 2. La fracción másica de la solución osmótica impregnada en la muestra ( $x$ ) por capilaridad o por el HDM y los valores de (De) en la fase líquida de la fruta.

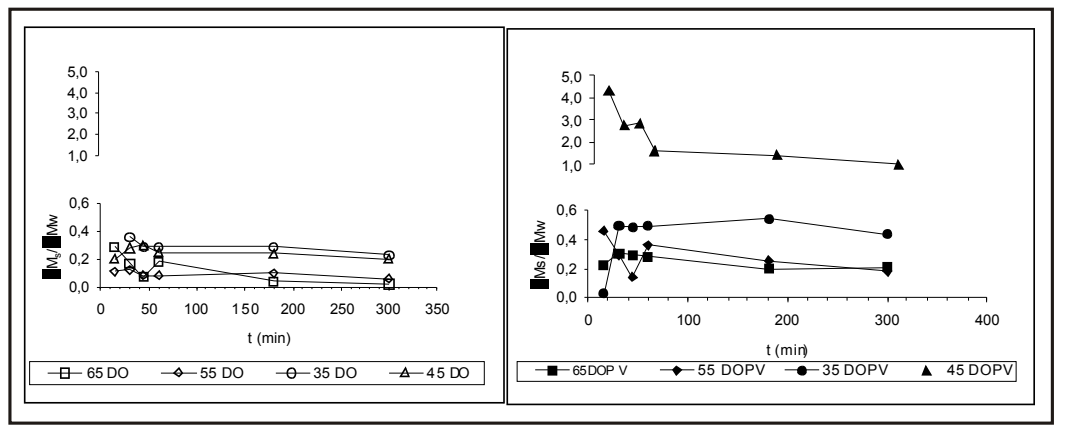

Figura 1. Evolución de la proporción de la ganancia de azúcar/ pérdida de agua, relacionada con los tiempos de proceso en los tratamientos DO y DOPV, realizados con soluciones de sacarosa a diferentes concentraciones

\section{Coeficiente de difusión (cambios de concentración en la fase líquida de fruta)}

Los valores del coeficiente de difusión efectiva (De) de los tratamientos DO y DOPV (Tabla 2) fueron obtenidos de la relación lineal entre 1 Y y la raíz cuadrada del tiempo de proceso(ecuación 3).
En todos los casos la De se incrementó cuando la concentración de la 50 disminuyó en los procesos DO y DOPV. Así mismo, los valores en los tratamientos DOPV fueron más altos que en los tratamientos DO para una so de concentración determinada, tal como se ha observado para otras frutas (Fito, P. \& Chiralt, A. 2000; Barat J., Fito P., \& Chiralt A. 2001b)

\section{Estudio de equilibrio}

El estudio del equilibrado se realizó en dos etapas: en la primera se analizó el equilibrio composicional, donde se determinó el contenido de humedad y de sólidos solubles en las muestras y en la solución, además de la $a_{w}$. En la segunda etapa se evaluaron los cambios estructurales ocurridos en las muestras durante la etapa de relajación de la matriz celular de la 
la fruta, determinando la evolución de la masa y volumen de las muestras durante un periodo de tiempo más largo (720 horas).

\section{Equilibrio composicional}

En la figura (2) se muestra, respectivamente, los valores de la actividad del agua $\left(a_{w}\right)$ y la fracción másica de la fase líquida (grados Brix / 100) de la matriz sólida $\left(z_{\mathrm{s}}\right)$ graficados en función del tiempo. Además, se presenta (en líneas discontinuas) la evolución de la fracción másica de solutos $\left(Y_{\mathrm{s}}\right)$ de la solución y la $a_{w}$ de cada una de las 50.
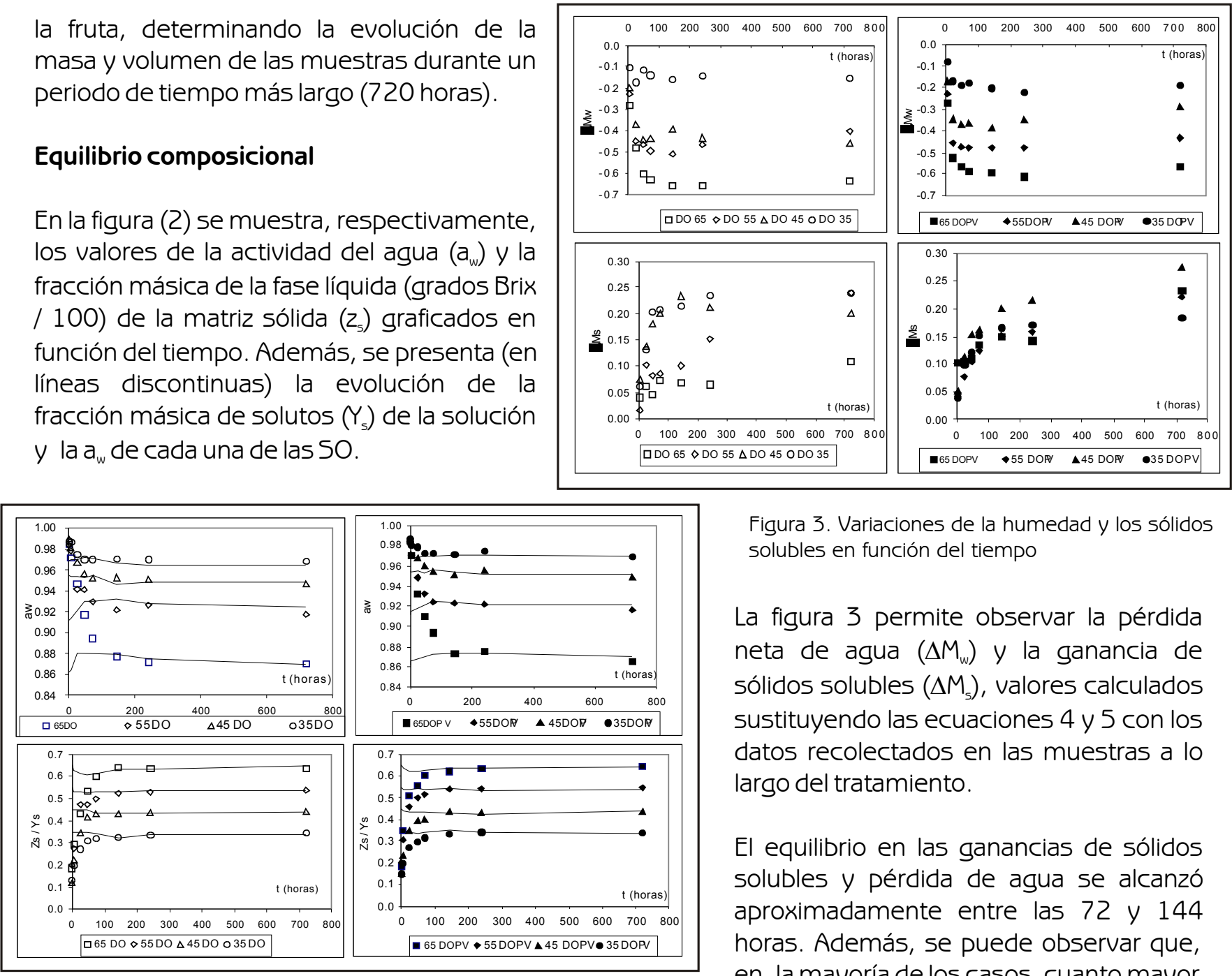

Figura 3. Variaciones de la humedad y los sólidos solubles en función del tiempo

La figura 3 permite observar la pérdida neta de agua $\left(\Delta M_{w}\right)$ y la ganancia de sólidos solubles $\left(\Delta M M_{s}\right)$, valores calculados sustituyendo las ecuaciones 4 y 5 con los datos recolectados en las muestras a lo largo del tratamiento.

El equilibrio en las ganancias de sólidos solubles y pérdida de agua se alcanzó aproximadamente entre las 72 y 144 horas. Además, se puede observar que, en la mayoría de los casos, cuanto mayor

Figura 2. Cambios en la actividad de agua $\left(a_{w}\right)$ y en la fracción másica de sólidos solubles $\left(z_{\mathrm{s}}\right)$ de las muestras con respecto a la solución osmótica.

Se pudo apreciar que para los distintos casos, la igualdad en los valores de $a_{w}$ de las muestras y las soluciones osmóticas ocurrió alrededor de las 72 horas de tratamiento y un poco más tarde para las muestras que fueron deshidratadas con soluciones a $65^{\circ}$ Brix. Es destacable que la igualdad de $\mathrm{a}_{\mathrm{w}}$ se estabilizó después de las $72 \mathrm{~h}$ entre muestras y soluciones. Una de las causas de este comportamiento puede estar relacionada con la integridad y la actividad biológica de la estructura vegetal que se conservó todo el tratamiento. es la concentración de la solución osmótica menor es la ganancia de sólidos y mayor es la pérdida de agua en la muestra, lo que contribuye a que tarde más en alcanzar la estabilidad. Al comparar las muestras tratadas por DO y DOPV en la variación $\Delta \mathrm{m}_{\mathrm{w}}$, se aprecian leves diferencias entre ellas, con unas pérdidas de agua ligeramente inferiores en el último caso, debido a que las muestras tratadas por DOPV sustituyeron el gas ocluido por solución osmótica. En cuanto a $\Delta M_{s}$ se apreció una diferencia muy marcada en el tratamiento a $45^{\circ}$ Brix, lo que muestra una fuerte ganancia de sólidos en DOPV. 


$$
\begin{aligned}
& \Delta \mathrm{M}_{\mathrm{W}}=\frac{\left(\mathrm{M}^{\mathrm{t}} \mathrm{x}_{\mathrm{w}}^{\mathrm{t}}\right)-\left(\mathrm{M}^{0} \mathrm{x}_{\mathrm{w}}^{0}\right)}{\mathrm{M}^{0}} \\
& \Delta \mathrm{M}_{\mathrm{S}}=\frac{\left(\mathrm{M}^{\mathrm{t}} \mathrm{x}_{\mathrm{ss}}^{\mathrm{t}}\right)-\left(\mathrm{M}_{0} \mathrm{x}_{\mathrm{ss}}^{0}\right)}{\mathrm{M}^{0}}
\end{aligned}
$$

Donde:

$M^{t}=$ masa en tiempot $M^{\circ}=$ masa inicial

$X_{w}^{\circ}=$ humedad inicial $x_{w}^{t}=$ humedad en tiempo $t$

$x_{s s}^{\circ}=$ sólidos solubles iniciales $x_{s s}^{t}=$ sólidos

solubles en tiempo $t$

\section{Evolución de la masa y volumen}

La figura 4 presenta las variaciones de masa $(\Delta M)$ y volumen $(\Delta V)$, calculados en las ecuaciones 1 y 2 , con los datos obtenidos en las muestras sometidas a deshidratación osmótica durante largos periodos de tiempo (hasta 720 horas).

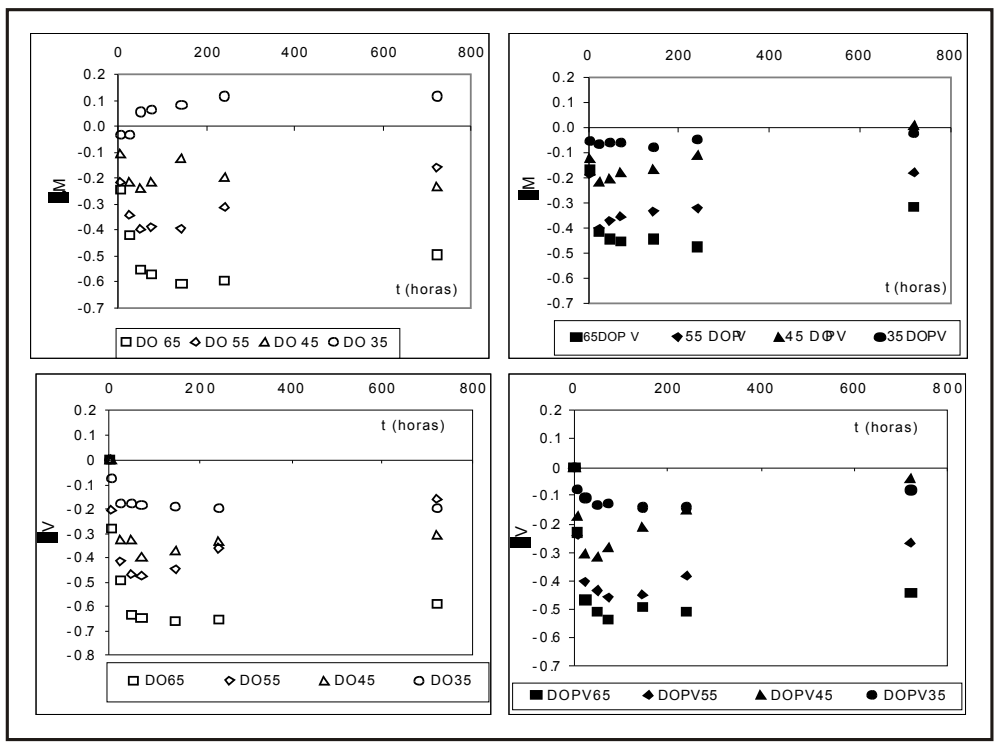

Figura 4. Variaciones de masa y volumen en función del tiempo.

A las primeras 72 horas, la mayoría de los tratamientos presentaron pérdidas de masa y volumen. Transcurrido este tiempo, se observó en las muestras una recuperación (excepto la de 35ํix). Este comportamiento se encuentra asociado con la relajación de la matriz celular contraída por la deshidratación (Fito P. \& Chiralt A. 2000; Barat J., Chiralt A., \& Fito P. 1998); dicha relajación conduce a la recuperación de volumen y masa debido a la succión de solución externa.

El estudio de la variación de masa y volumen durante el equilibrado osmótico de mango, muestra que los tratamientos con $\mathrm{SO}$ a baja concentración fomentan la ganancia de la solución externa en las muestras. Este efecto se debe a la viscosidad de la solución asociada a la capilaridad de la matriz sólida, también se relaciona con el efecto del mecanismo hidrodinámico en los poros del tejido, mientras que en los tratamientos con 50 a altas concentraciones no se presentan ganancias significativas en tratamientos prolongados por la escasa recuperación de volumen asociada a deformaciones que provocan la no elasticidad de la matriz celular durante la deshidratación, debido probablemente a los fenómenos de encostramiento; un comportamiento similar reportan en otras frutas Barat et al., (2000) y Talens (2002). Esto muestra la bondad del tratamiento con $45^{\circ}$ Brix, que ofrece un balance positivo en la variación de agua y solutos, adecuado para la obtención de productos escarchados, donde el volumen y la textura del producto terminado son altamente valorados.

\section{Cambios estructurales}

En esta sección se analiza la evolución del volumen de las muestras durante los tratamientos osmóticos observados hasta mucho después del equilibrado composicional de las muestras (720 horas de tratamiento), con el propósito de analizar los fenómenos de relajación del volumen observados en los tejidos vegetales, responsables de la ganancia de masa a tiempos largos de tratamiento (Barat J., Chiralt A., \& Fito P. 1998; Barat J. et al., 1999; Fito P. et al., 2001). 


\section{Cambios de volumen}

Para explicar los cambios de volumen de las muestras durante los tratamientos osmóticos, se ha comparado el cambio de volumen medido experimentalmente $\left(\Delta \mathrm{V}_{\mathrm{T}}\right)$ con el cambio de volumen de la fase liquida de la fruta $\left(\Delta \mathrm{V}_{\mathrm{FL}}\right)$. Este último se calculó sustituyendo en la ecuación 6 y los datos de composición de la muestra fresca y tratada, así como la densidad inicial de la muestra $\left(\rho_{0}\right)$ y de la fase líquida de la fruta $\left(\rho_{\mathrm{FL}}\right)$. El cálculo de la densidad de la fase líquida de la fruta se realizó a partir de la concentración de la fase líquida $\left(z_{\mathrm{s}}\right)$, mediante la ecuación 7 , que fue obtenida del ajuste $\left(r^{2}=0.999\right)$ de los datos experimentales de la relación densidad: concentración para diferentes azúcares y polioles en el intervalo de $Z_{s}$ entre 0 y 0.8 (Lewis M., 1996).

$\Delta \mathrm{V}_{\mathrm{FL}}=\frac{\frac{\mathrm{m}^{\mathrm{t}}\left(\mathrm{x}_{\mathrm{w}}^{\mathrm{t}}+\mathrm{x}_{\mathrm{s}}^{\mathrm{t}}\right)}{\rho_{\mathrm{FL}}^{\mathrm{t}}}-\frac{\mathrm{m}^{0}\left(\mathrm{x}_{\mathrm{w}}^{0}+\mathrm{x}_{\mathrm{s}}^{0}\right)}{\rho_{\mathrm{FL}}^{0}}}{\frac{\mathrm{m}^{0}}{\rho^{0}}}$

$\rho_{\mathrm{FL}}=230 Z_{\mathrm{s}}^{2}+339 Z_{\mathrm{s}}+1000$

Los valores de $\Delta \mathrm{V}_{\mathrm{FL}}$ (ecuación 8) se calcularon de forma independiente a partir de la pérdida volumétrica de agua $\left(\Delta \mathrm{V}_{w}\right)$ y la ganancia volumétrica de solutos $\left(\Delta v_{s}\right)$, mediante las ecuaciones 9 y 10 y se compararon con los valores obtenidos en la ecuación 6. La figura 5 muestra la concordancia de los valores obtenidos para todos los casos, lo que apoya la fiabilidad de los procedimientos.
$\Delta \mathrm{V}_{\mathrm{FL}}=\Delta \mathrm{V}_{\mathrm{w}}+\Delta \mathrm{V}_{\mathrm{s}}$
$\Delta \mathrm{V}_{\mathrm{w}}=\frac{\Delta \mathrm{M}_{\mathrm{w}} \rho_{0}}{\rho_{\mathrm{w}}}$
$\Delta \mathrm{V}_{\mathrm{s}}=\frac{\Delta \mathrm{M}_{\mathrm{s}} \rho_{0}}{\rho_{\mathrm{s}}}$

Donde: $\rho_{0}=$ densidad inicial de la muestra en $\mathrm{kg} / \mathrm{m}^{3} ; \rho_{\mathrm{w}}=1000 \mathrm{~kg} / \mathrm{m}^{3} ; \rho_{\mathrm{s}}=1590 \mathrm{~kg} / \mathrm{m}^{3}$

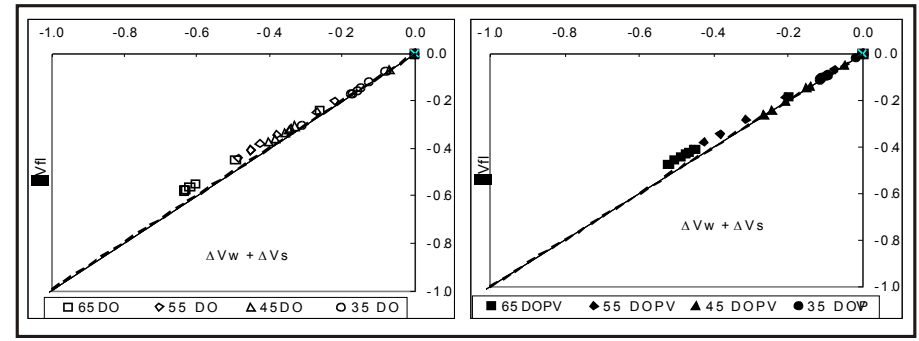

Figura 5. Comparación entre las variaciones de volumen de la fracción líquida de las muestras $\left(\Delta V_{F L}\right)$ obtenidas por dos procedimientos diferentes

El cambio de volumen de las muestras durante los tratamientos osmóticos puede ser explicado haciendo uso del modelo descrito por Fito P. et al., (2002), donde demostró que el cambio total de volumen es equivalente a la suma de los cambios en cada una de las 3 fases idealizadas del producto: Fase gas $\left(\Delta \mathrm{V}_{\mathrm{FG}}\right)$, fase líquida (agua más solutos) $\left(\Delta \mathrm{V}_{\mathrm{FL}}\right)$ y matriz sólida (insolubles) $\left(\Delta \mathrm{V}_{\mathrm{MS}}\right)$ (ecuación 11). La fracción de insolubles representa un volumen muy pequeño en comparación con las otras dos fases y puede asumirse que cambia relativamente poco a lo largo del proceso. Los cambios ocurridos en la fase gas y líquida son debidos a la salida e ingreso de agua y solutos por los diferentes mecanismos y al cambio de volumen del gas de los poros, en el cual los cambios de presión en el sistema tienen una gran importancia. Para muestras muy poco porosas $\Delta \mathrm{V}_{\mathrm{FG}}$ es prácticamente cero y la variación de volumen de las muestras viene explicada por la variación de volumen de la fase líquida en los tratamientos DO y DOPV (Fito P. et al., . 2002).

$$
\Delta \mathrm{V}=\Delta \mathrm{V}_{\mathrm{FL}}+\Delta \mathrm{V}_{\mathrm{FG}}+\Delta \mathrm{V}_{\mathrm{MS}}
$$

La figura 6 muestra las variaciones de volumen total $(\Delta \mathrm{V})$, volumen de la fase gas $\left(\Delta \mathrm{V}_{\mathrm{FG}}\right)$ y volumen de la fase liquida $\left(\Delta \mathrm{V}_{\mathrm{FL}}\right)$ del mango en los tratamientos DO y DOPV. 


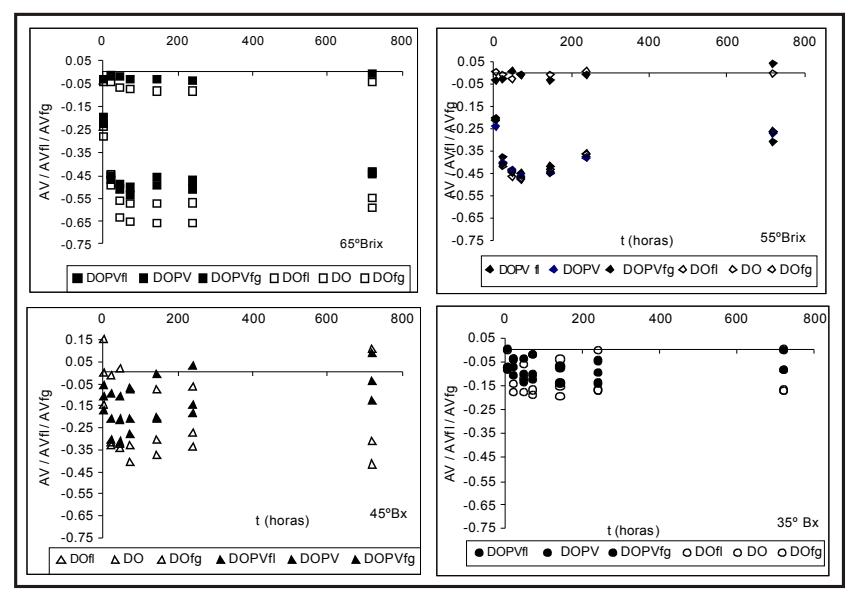

Figura 6. Variación de volumen total $(\Delta V)$, de la fase gas $\left(\Delta \mathrm{V}_{\mathrm{FG}}\right)$ y de la fase liquida $\left(\Delta \mathrm{V}_{\mathrm{FL}}\right)$ del mango para los distintos tratamientos.

Las muestras tratadas con soluciones $45 \mathrm{y}$ 55०Brix, al contrario de las tratadas con 35 y $65^{\circ}$ Brix presentan un gran poder de relajación. En cuanto a los tratamientos DOPV, estos recuperan su volumen en mayor medida.

\section{Escarchado: variaciones en la masa, humedad y sólidos solubles}

El trabajo de escarchado se realizó en dos etapas. La primera comprendió el pretratamiento de deshidratación osmótica, y la segunda del secado con aire caliente. A continuación en la tabla 3 se presentan los valores de humedad, ${ }^{\circ B r i x ~ y ~ d e ~}$ contenido en sólidos solubles del producto, determinados por la ecuación 12, alcanzado al término del pretratamiento osmótico en cada caso; también se da la variación de masa.

$x_{s s}^{t}=\frac{Z_{s}^{t} * X_{w}^{t}}{1-Z_{s}^{t}}(12) \quad$ donde: $Z_{s}^{t}=\frac{{ }^{o} \text { Brix }}{100}$

Donde :

$x_{s s}^{t}=$ fracción de sólidos solubles en un tiempo $t$

$x_{w}^{t}=$ fracción de humedad en un tiempo $t$

$z_{s}^{t}=$ grados Brix/100 en un tiempo $t$

Puede observarse que los ${ }^{\circ}$ Brix alcanzados fueron muy similares a los de la solución osmótica correspondiente, tal como se esperaba para el tiempo de equilibrado, determinado en 72 horas, en trabajos previos. Los contenidos en humedad y sólidos solubles fueron similares entre tratamientos. Las variaciones de masa al final del pretratamiento osmótico fueron mayores con soluciones de mayor concentración y es el resultado de la potenciación de la ganancia de solutos frente a la pérdida de agua, a medida que aumenta la concentración / viscosidad de la disolución.

La tabla 4 presenta los valores de humedad, sólidos solubles, ${ }^{\circ B r i x ~ y ~ v a r i a c i o ́ n ~ d e ~ m a s a ~}$ alcanzados en la etapa de secado por las muestras a los niveles de 68 y $72^{\circ}$ Brix.

Tabla 3. Humedad $\left(x_{w}{ }^{p q}\right)$, Sólidos solubles $\left(x_{s s}{ }^{p q}\right)$, ${ }^{\circ}$ Brix $y$ variación de masa al final del pretratamiento osmótico de los diferentes tratamientos de cilindros de mango.

\begin{tabular}{|ccccc|}
\hline Muestra & ${ }_{x w}{ }^{P O}$ & ${ }_{x s s}{ }^{P O}$ & ${ }^{\circ}$ Brix & \\
\hline & & & & $\Delta M P O$. \\
2565 & 0.35 & 0.59 & 63.0 & -0.3900 .204 \\
3565 & 0.35 & 0.62 & 64.2 & $-0.423 \pm 0.126$ \\
4545 & 0.57 & 0.38 & 39.7 & $-0.211 \pm 0.070$ \\
4565 & 0.32 & 0.58 & 64.8 & $-0.449 \pm 0.089$ \\
5565 & 0.35 & 0.60 & 63.1 & $-0.511 \pm 0.031$ \\
6565 & 0.33 & 0.60 & 64.6 & $-0.506 \pm 0.057$ \\
\hline
\end{tabular}

Tabla 4. Humedad $\left(x_{w}{ }^{p O}\right)$, Sólidos solubles $\left(x_{s s}{ }^{p o}\right)$, grados Brix ('Brix) y variación de masa al final de la etapa de secado de los diferentes tratamientos de los cilindros de mango.

\begin{tabular}{|c|c|c|c|c|}
\hline \multicolumn{5}{|c|}{$68^{\circ}$ Brix } \\
\hline Muestra & $\mathrm{X}_{\mathrm{w}}^{\mathrm{f}}$ & $\mathrm{X}_{\mathrm{ss}}^{\mathrm{f}}$ & ${ }^{\circ}$ Brix $^{\mathrm{f}}$ & $\Delta \mathrm{M}_{\mathrm{f}}$ \\
\hline 2565 & $0.306 \pm 0.014$ & $0.630 \pm 0.012$ & $67.3 \pm 1.3$ & $-0.320^{ \pm} 0.019$ \\
\hline 3565 & $0.309 \pm 0.004$ & $0.661 \pm 0.004$ & $68.1 \pm 0.4$ & $-0.566 \pm 0.006$ \\
\hline 4545 & $0.313 \pm 0.051$ & $0.600 \pm 0.045$ & $65.8 \pm 5.4$ & $-0.473 \pm 0.192$ \\
\hline 4565 & $0.294 \pm 0.012$ & $0.601 \pm 0.010$ & $67.1 \pm 1.3$ & $-0.613^{ \pm} 0.010$ \\
\hline 5565 & $0.312 \pm 0.008$ & $0.634 \pm 0.008$ & $67.0 \pm 0.8$ & $-0.614 \pm 0.011$ \\
\hline 6565 & $0.314 \pm 0.010$ & $0.619 \pm 0.009$ & $66.3 \pm 1.0$ & $-0.637 \pm 0.014$ \\
\hline
\end{tabular}

\begin{tabular}{|c|c|c|c|c|}
\hline \multicolumn{5}{|c|}{72 Brix $^{\circ}$ (Promedios SAC y MW) } \\
\hline Muestra & $\mathrm{X}_{\mathrm{w}}^{\mathrm{f}}$ & $\mathrm{X}_{\mathrm{ss}}^{\mathrm{f}}$ & ${ }^{\circ}$ Brix $^{\mathrm{f}}$ & $\Delta \mathrm{M}_{\mathrm{f}}$ \\
\hline 2565 & $0.251^{ \pm} 0.011$ & $0.673^{ \pm} 0.010$ & $72.8^{ \pm} 1.1$ & $-0.465^{ \pm} 0.184$ \\
\hline 3565 & $0.269 \pm 0.004$ & $0.700 \pm 0.004$ & $72.2 \pm 0.4$ & $-0.485^{ \pm} 0.112$ \\
\hline 4545 & $0.297 \pm 0.039$ & $0.614 \pm 0.034$ & $67.5 \pm 4.1$ & $-0.510^{ \pm} 0.067$ \\
\hline 4565 & $0.243^{ \pm} 0.011$ & $0.644 \pm 0.009$ & $76.6 \pm 1.2$ & $-0.503^{ \pm} 0.080$ \\
\hline 5565 & $0.268^{ \pm} 0.016$ & $0.675^{ \pm} 0.015$ & $71.6 \pm 1.7$ & $-0.566^{ \pm} 0.032$ \\
\hline 6565 & $0.256 \pm 0.012$ & $0.672 \pm 0.011$ & $72.4 \pm 1.2$ & $-0.555^{ \pm} 0.054$ \\
\hline
\end{tabular}


Los valores medios de los oBrix fueron muy cercanos a los valores preestablecidos para cada uno de los tratamientos, excepto en al caso del tratamiento 4545 en que se presentó gran resistencia al final del secado, probablemente como consecuencia de los cambios composicionales ocurridos durante el tratamiento osmótico, donde la ganancia de azúcar fue muy significativa. Las variaciones de masa presentadas al final del escarchado en los tratamientos equilibrados a $65^{\circ}$ Brix en la etapa osmótica no presentan diferencias significativas entre sí, mientras que en los equilibrados 2565 y 4545 , las variaciones de masa fueron menores.

\section{Ensayos mecánicos}

La fuerza empleada en los ensayos de corte de los cilindros de mango secados por métodos combinados se muestran en la figura 7.

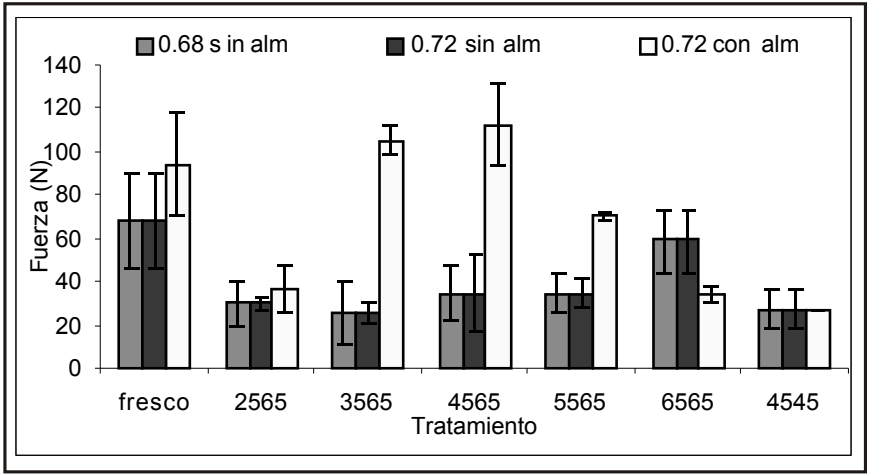

Figura 7. Fuerza de corte de los cilindros de mango a las diferentes concentraciones de soluciones osmóticas y diferentes procesos de secado.

Se observaron diferencias significativas en cuanto a la fuerza empleada para el corte de las muestras de los diferentes procesos, donde se presentaron como iguales los procesos de secado a 0.68 y 0.72 con aire caliente y no almacenados. Esto pudo ser causado por la poca diferencia en la concentración de las muestras al final del secado y por la poca disponibilidad de tiempo para cristalizar el azúcar. Al comparar los tratamientos 0.72 con y sin almacenamiento, se encontró que en la mayoría de las muestras almacenadas se ocasionó un endurecimiento, posiblemente por la cristalización de los azúcares. Al comparar muestras entre tratamientos y a un mismo nivel de proceso, se observaron diferencias entre ellas, lo que presentó desde productos muy blandos (4545) hasta otros con máxima dureza (fresco).

\section{Análisis sensorial}

A efectos de comparar sensorialmente las muestras de mango obtenidas por diferentes tratamientos, se realizaron dos pruebas. En la primera, se sometieron a análisis un total de 5 muestras; una se trató con calor por el sistema comercial y las restantes se escarcharon bajo la metodología propuesta. Se seleccionaron 10 jueces para evaluar la textura. El atributo de textura (dureza) se presenta en la figura 8.

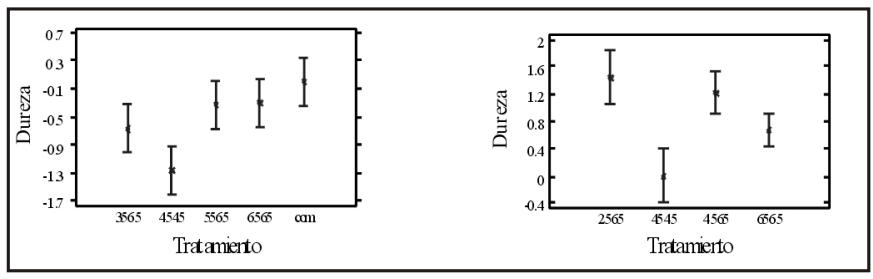

Figura 8. prueba sensorial de la textura en mango escarchado a temperatura moderada y pulso a vacío y escarchado comercial

Mo se presentaron diferencias estadísticas significativas entre la mayoría de los tratamientos, salvo en 4545, que mostró ser el más blando, mientras que el más duro fue el tratado con calor. Este comportamiento puede deberse al efecto causado por la viscosidad de la disolución en la estructura de la fruta para 4545 y a la temperatura para la comercial (com). Para validar este resultado se tomó como muestra patrón 4545 y se comparó con 2565, 4565 y 6565 en el mismo atributo. El resultado presenta una respuesta decreciente a medida que se aumenta la concentración de la disolución en el preproceso osmótico, se confirmó que la muestra patrón (4545) era la menos dura de todas.

\section{CONCLUSIONES}

$\square$ El tratamiento de deshidratación osmótica en disoluciones de $45^{\circ}$ Brix, con pulso a vacío, se presentó como el más adecuado para deshidratar mango. 
$\square$ El tiempo del equilibrado osmótico durante la deshidratación se determinó en 72 horas.

■ La textura evaluada sensorialmente en las muestras escarchadas con tratamiento osmótico 4545 fue significativamente más gomosa, menos dura y más jugosa que el resto de las escarchadas a vacío. Estas muestras fueron, además, preferidas significativamente por losjueces.

$\square$ El tratamiento realizado con disolución a $45^{\circ}$ Brix y pulso a vacío, y secado hasta 72ºrix, se recomienda como el más apropiado para la producción de mango escarchado.

\section{REFERENCIAS}

$\square$ A.O.A.C. (1980). Association of official Analytical Chemist Official Methods of Analysis. Washington. D.C.

■ Argaiz A., López-Malo A., Palou E. \& Welti J. (1994). Osmotic dehydration of papaya with corn syrup solids. Drying Technology. 12(7): 1709-1725.

■ Barat, J., Chiralt, A., \& Fito, P. (1998). Equilibrium in cellular food-osmotic systems: The role of the structure. Journal Food Science. 63(5) 836-840.

$\square$ Barat J., Albors A., Chiralt A. \& Fito P. (1999). Equilibration of apple tissue in osmotic dehydration. Microestructural changes. En Drying '98. Ed. C.B. Akritidis; D. MarinosKouris y G.D. Saravacos. Ziti Editions, Thessloniki, Volumen A.: 827-835.

■ Barat J., González G., Chiralt A. \& Fito P. (2000) Vacuum Impregnation applied to pineapple candying. In J. Welty-Chanes, G.V. Barbosa-Cánovas, \& J.M. Aguilera Proceedings of the Eighth International Congress on Engineering and Food -ICEF 8(Pp.1372-1376). Lancaster: Technomic Publishing Co., Inc.

$\square$ Barat J., Fito P., \& Chiralt A. (2001a) Effect of osmotic solution concentration temperature and vacuum impregnation pre-treatment on osmotic dehydration kinetics of apple slices.
Journal Food science and technology international. 5 (7) 451-456.

$\square$ Barat J., Fito P., \& Chiralt A. (2001b). Modelling of simultaneous mass transfer and structural changes in fruit tissues. Journal of Food Engineering, 49 (2-3), 77-85

$\square$ Barat J., Talens P., Barrera C. \& Chiralt A. (2002). Mo-thermal pineapple candying optimisation. Journal Food Science. 86(4) 636-641.

$\square$ Crank J. (1975). The mathematics of the diffusion. Oxford: Clarendon Press.

v FAO (2000). Comité de problemas de productos básicos subgrupo sobre frutas tropicales. Primera reunión Pattaya, Tailandia, 25 - 28 de mayo de 1998. Home Page, http://www.fao.org

$\square$ Fito P., Andrés A., Chiralt A. \& Pardo P. (1996). Coupling of Hydrodynamic Mechanism and Deformation Relaxation Phenomena during vacuum treatments in solid porous food liquid systems. Journal of Food Engineering. 27 (3) 229240.

$\square$ Fito P. \& Chiralt A. (1997). Osmotic dehydration. An approach of the modelling of solid food liquid operations. P. Fito, E. OrtegaRodriguez, and G.V. Barbosa-Cánovas (ed.). In Food Engineering 2000, Ch 13 (pp. 231252). Chapman and Hall, Mew York.

$\square$ Fito P. \& Chiralt A. (2000). Vacuum impregnation of plant tissues. In: Design of minimal processing technologies for fruit and vegetables. Ed. S.M., Alzamora, M.S., Tapia, \& A. López-Malo, Aspen Publishers, Inc. Maryland. (pp. 189-205).

$\square \quad$ Fito P., Betoret M., Gras M., Martínez-Monzó J. \& Chiralt A. $\left(2001^{a}\right)$ Viability of vegetables matrices as support of physiological active components. Proceedings of ICEF \& Technomic Publishing co., Inc. Lancaster, volumen II, 1366-1371.

■ Fito P., Chiralt A., Barat J. \& Martínez-Monzó J. (2002). Mass transport and deformation relaxation phenomena in plant tissues. Ed. J Welti-Chanes, G. V. Barbosa-Canovas, J. M. Aguilera. Engineering and Food for the 21st Century. Lancaster. Boca Ratón: CRC Pres. 
Pág. 235-254.

$\square$ Gil A., Duarte I., Delgadillo I., Colquhoun J., Casuscelli F., Humper E., \& Spraul M. (2000). Study of the Compositional Changes of Mango During Ripening by Use of Muclear Magnetic Resonance Spectroscopy. Journal Agric. Food Chem. 48 (5) 15241536.

■ Giraldo G., Talens P., Fito P. \& Chiralt A. (2003) Influence of sucrose solution concentration on kinetics and yield of mango osmotic dehydration. Journal of Food Engineering (58) 33-43.

$\square$ Lewis M. (1996). Physical properties of foods and food processing systems. Chichester: Ellis Harwood Ltd.

v Maldonado 5. \& Sammán M(2000). Dehydration of tropical fruits produced in noa region, Argentina: Mango (Mangifera indica) stage 1. In J. Welty-Chanes, G.V. BarbosaCánovas, \& J.M. Aguilera Proceedings of the Eighth International Congress on Engineering and Food -ICEF 8- (pp.1204-1208). Lancaster: Technomic Publishing Co., Inc.

$\square$ Mujica-Paz H., Valdez-Fragoso A, MartínezPantoja Y \& Welti_Canes J. (2000) Response surface methodology applied to vacuum osmotic dehydration of mango slices. In $J$. Welty-Chanes, G.V. Barbosa-Cánovas, \& J.M. Aguilera Proceedings of the Eighth International Congress on Engineering and Food -ICEF 8- (PP.1305-1308). Lancaster: Technomic Publishing Co., Inc.

ø Ribeiro M. \& Anuncio De Sabaa A. (2000). Mango(Mangifera indica L.) saturación del cV. Rosa con azúcares. Ciencia e Tecnología de Alimentos; 19 (1) 118-122.

■ Talens P., (2002) Tratamientos osmóticos en la crioprotección de fresa y kiwi. Tesis Doctoral. Universidad Politécnica de Valencia. España.

■ Vial C., Gulibert S. \& Cuq J. (1991). Osmotic Dehydration of Kiwi Fruits: Influence of process variables on the colour and ascorbic acid content. Sciences des Aliments, (11): 63-64. 